\title{
Review: behavioural interventions plus laxatives are effective for defaecation disorders, but biofeedback does not add benefit
}

Source of funding: National Health Service.

For correspondence: Ms M Brazzelli University of Aberdeen, Aberdeen, Scotland, UK. mgb@hsru.abdn.ac.uk.

A modified version of this abstract also

appears in

Evidence-Based

Nursing.

Unsuccessful rates for cognitive and behavioural interventions for child defecation disorders*

\begin{tabular}{|c|c|c|c|c|}
\hline Comparisons & Follow up & $\begin{array}{l}\text { Weighted } \\
\text { event rates }\end{array}$ & RRI $(95 \%$ CI) & NNH (Cl) \\
\hline \multirow{2}{*}{$\begin{array}{l}\text { Conv }+ \text { biofeed } v \\
\text { conv }\end{array}$} & 12 months & $52 \% \vee 45 \%$ & $16 \%(-4$ to 40$)$ & Not significant \\
\hline & 18 months & $52 \% \vee 46 \%$ & $15 \%(-11$ to 48$)$ & Not significant \\
\hline \multirow{3}{*}{$\begin{array}{l}\text { Biofeed }+ \text { lax } v \\
\text { biofeed }\end{array}$} & 12 weeks & $91 \%$ v 56\%† & $63 \%$ (16 to 149$)$ & 3 (2 to 10$)$ \\
\hline & 12 months & $91 \%$ v 64\%† & $43 \%(5.3$ to 108$)$ & 4 (3 to 28$)$ \\
\hline & & & RRR (Cl) & NNT (Cl) \\
\hline \multirow{2}{*}{$\begin{array}{l}\text { B-mod + lax } v \\
\text { B-mod }\end{array}$} & 6 months & $43 \%$ v $60 \%$ & $28 \%$ (5 to 46$)$ & 6 (4 to 28$)$ \\
\hline & 12 months & $36 \%$ v $51 \%$ & $30 \%$ (3 to 49$)$ & $7(4$ to 48$)$ \\
\hline B-mod + lax $v$ lax & Not reported & $15 \%$ v $55 \% \dagger$ & $73 \%$ (36 to 90$)$ & 3 (2 to 7$)$ \\
\hline
\end{tabular}

${ }^{*}$ Biofeed $=$ biofeedback; B-mod $=$ behavioural modifications; conv = conventional treatment (laxatives, dietary advice, and toilet training); lax = laxatives. Other abbreviations defined in glossary; RRI, RRR, NNH, NNT, and Cl calculated from data in article. †Event rates not weighted.

\section{COMMENTARY}

Encopresis interferes with normal social and psychological development and strains family relationships. Brazzelli and Griffiths have done a great service with this systematic review of one important aspect of the management of this condition. This review provides some evidence that conventional treatment works, although that was not its primary purpose. The combination of B-mod plus laxatives is more effective than B-mod alone or laxatives alone. This finding should be of some comfort to those who treat children with this condition, but the authors point out the appalling lack of scientific evidence about each component of conventional treatment, including the relative efficacy of various types and doses of laxative.

The main new piece of information from this review is the conclusion that biofeedback adds nothing to conventional treatment. Biofeedback trains children to tighten and relax their perineal muscles and increase the efficiency of defaecation. This review suggests that even when that objective is achieved, it does not correlate with successful resolution of encopresis. In fact, there seems to be a trend towards worse outcomes with biofeedback, and when the one study that was strongly positive and accounted for the heterogeneity was removed, the outcomes were worse. This finding needs further explanation and study. Is our understanding of the mechanism of this condition incorrect? Or is there a great deal of variability in the skill and manner in which biofeedback was used? The clinical bottom line is that widespread adoption of biofeedback has no role in the care of children with this condition.

The balancing of the specific components of conventional treatment (eg, laxatives, diet, education, and stress reduction) in specific families remains very much an art rather than a science. A review directed at conventional treatment is indicated but will almost certainly leave many unanswered questions. Since encopresis is not a rare condition, these questions can be addressed with carefully designed studies.

Michael E Moffatt, MD University of Manitoba Winnipeg, Manitoba, Canada
Centre Register) in March 2001, scanning reference lists, and contacting experts.

\section{Study selection}

Studies were selected if they were randomised or quasirandomised controlled trials of cognitive, behavioural, or cognitive and behavioural interventions in children with a history of faecal soiling with or without constipation.

\section{Data extraction}

The quality of studies was assessed. Data were extracted on participant characteristics, interventions, and outcomes.

\section{Main results}

14 studies (12 randomised controlled trials, 843 children) met the selection criteria. Children had encopresis (ie, inappropriate passage of stool in children $>4 \mathrm{y}$ of age) in 12 studies and faecal incontinence resulting from congenital abnormalities in 2 studies. Study duration ranged from 2 weeks to 12 months. 8 studies compared conventional treatment (laxatives, dietary advice, and toilet training) plus biofeedback with conventional treatment alone. The groups did not differ for the number of children who were not cured or improved at 12 or 18 months (table). 1 study compared biofeedback training plus laxatives with biofeedback alone. More children in the biofeedback plus laxatives group than in the biofeedback alone group were not cured or improved at 12 weeks and 12 months (table). 2 studies compared B-mod plus laxatives with B-mod alone. The laxative group had fewer unsuccessful children than did the B-mod alone group at 6 and 12 months (table). 1 study compared B-mod (diet modifications and scheduled toileting) with mineral oil (laxative); groups did not differ for any outcomes. 1 study compared B-mod plus psychotherapy with B-mod alone; treatment results were similar in both groups. 1 study compared 3 groups: B-mod (incentive programmes and toilet training) plus laxatives, laxatives alone, and biofeedback. B-mod plus laxatives were better than laxatives alone for reducing the number of children who were not cured or improved (table).

\section{Conclusions}

In children with defaecation disorders, biofeedback does not add benefit to conventional treatment. The combination of behavioural modification techniques and laxatives is more effective than either intervention alone. 